\title{
Biprism Method of Determining the Equivalent Focal Length of Flat Field Lenses
}

\author{
Walter R. Darling
}

(June 25, 1962)

\begin{abstract}
A device is described that permits the rapid determination of the equivalent focal length of a lens. A transmitting biprism mounted between a collimated light source and a lens, divides the light incident upon the front of the lens into two parallel beams making a fixed angle with one another. On passing through the lens, two images are formed in the focal plane. The magnitude of the lateral separation of the images is determined by the angular separation of the two incident beams and the focal length of the lens. The focal length of the imaging lens may be determined from the measured separation of the images at the focal plane of the lens and the known angle of deviation of the two incident beams produced by the biprism.
\end{abstract}

\section{Introduction}

The National Bureau of Standards is frequently called upon to determine the equivalent focal length and related constants of lenses with a high degree of accuracy. For many types of lenses these measurements are customarily performed visually on the precision optical bench to within $\pm 0.10 \mathrm{~mm}$ on lenses whose equivalent focal lengths range up to $200 \mathrm{~mm}, \pm 0.15 \mathrm{~mm}$ for lenses whose equivalent focal lengths range between 200 and $800 \mathrm{~mm} . \pm 0.25$ $\mathrm{mm}$ for lenses whose equivalent focal lengths range from 800 to $1,200 \mathrm{~mm}$, and $\pm 0.50 \mathrm{~mm}$ for lenses whose equivalent focal lengths range from 1,200 to $1,800 \mathrm{~mm}^{1}$

Frequently it is desirable to determine only the equivalent focal length of lenses for use in evaluation of certain metrical qualities, such as distortion and resolution at finite and infinite distances. For these determinations the values of the focal lengths need not be as accurately known as those obtained by the precision optical bench method, yet it is desirable to know these values to a greater degree of accuracy than the value marked on the front of the lens, which is referred to as the nominal focal length. The tolerance for the nominal focal length is given by the ASA Standards ${ }^{2}$ as \pm 4 percent.

There is small likelihood of gross error in optical bench methods, but to preclude the possibility of such error it is desirable to have a method that permits a quick check of the value of the equivalent focal length of a given lens obtained with the optical bench. It is moreover desirable that the check measurement be based upon a different principle of measurement and employing different data to minimize the danger of systematic error. In this paper a simple check method for measuring the equivalent focal length of a lens is presented. A biprism placed in front of the lens under test splits the incoming collimated beam into two parallel beams with a

\footnotetext{
I The differences in accuracy depend more upon the relative apertures than upon focal lengths, as a consequence of the differences in depth of focus.

2 ASA Standards-Focal Length Marking of Lenses. PH.3.13-1958.
}

fixed angular separation. With this prism, values of focal length accurate to within \pm 1 percent may be obtained. While the range of uncertainty is somewhat higher than that attained by the optical bench method it nonetheless permits one to evaluate the equivalent focal length of a given lens with a degree of precision well within the tolerances set forth in the ASA Standards for nominal focal length.

\section{Description of the Instrument}

An optical wedge of borosilicate optical glass approximately $2 \frac{3}{4}$ in. in diameter was ground and polished with an angle of approximately 2 degrees between the faces. The finished wedge was then cut on a diamond saw in the principal section across its diameter. One half of the wedge was inverted with respect to the remaining half so that the deviations were in opposite directions. These two halves were then cemented to a flat base of ground and polished optical glass of approximately $3 \frac{1}{8}$ in. in diameter and $1 / 2$ in. in thickness. For small wedge angles $\alpha$, the deviation angle $\theta$ for normal incidence is :

$$
\theta=(n-1) \alpha,
$$

$n$ being the index of refraction of the material used for the biprism. For this borosilicate glass $(n-1)$ $=0.519$. For a collimated beam of light incident normally upon one side of the biprism, the angle separating the two emergent beams is $2 \theta$. The magnitude of the angular deviation, $2 \theta$, was measured in the refractometry laboratory with white light and was found to be 2.059 degrees. The probable error of this measurement is approximately 6 sec. The rest of the instrument consists of a cell to hold the biprism and a rod attached to the cell for adjustment of the entire unit. The component parts of the instrument are illustrated in figure 1.

The wedge is not achromatic but the error resulting from the lack of achromatism is negligible. When white light passes through a prism (fig. 2), the components of different wavelengths are deviated by 


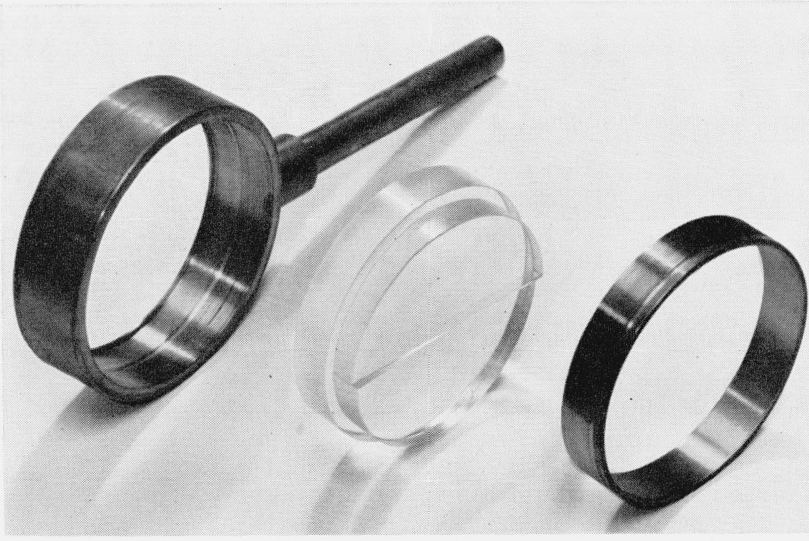

FIGURE 1. The component parts of the instrument are illustrated.

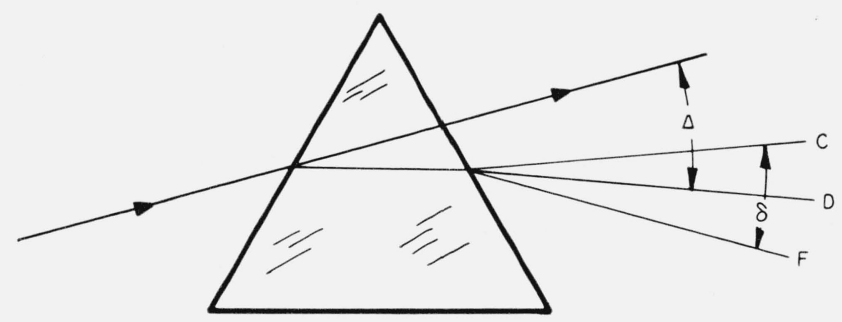

$$
\delta=\frac{\Delta}{64.5},=64.5
$$

Figure 2. When a white light beam passes through the wedge, the difference in the deviation of the red beam and the blue beam forms an angle $\delta$.

different amounts. The magnitude of the dispersion ir customarily measured by the difference between the deviations for red light, hydrogen 656 , and blue light, hydrogen 486 . Let this difference in deviation be called $\delta$. It may easily be deduced from eq (1) that

$$
\delta=\frac{\theta}{\nu}
$$

where $\nu$ is the Abbe number of the glass. Our biprism is made of borosilicate glass, $\nu=64.5$. Consequently

$$
\delta=0.015 \theta \text {. }
$$

The wavelength of maximum visibility, for which visual settings are made when white light is used, falls almost midway between the wavelengths $\mathrm{F}$ and $\mathrm{C}$. Since light at $\mathrm{F}$ and at $\mathrm{C}$ is definitely colored and also considerably less bright than that of maximum visibility, it is very unlikely that a setting would be off as far as $1 / 2 \delta$. Further, the deviation of the biprism consists of deviation to the left of $\theta$ plus deviation to the right of $\theta$. Consequently the dispersion is also half in one image and half in the other.

The error in setting, on one of the images, resulting from chromatic aberration should not exceed $\frac{1}{4} \frac{\theta}{\nu}$, where $2 \theta$ is the total deviation of both prisms, and the error in the measured separation of the two images from this source then should not exceed $1.4 x$

$\overline{(4)(64.5)}$, where $x$ is the separation of the images, or approximately one quarter of 1 percent of the measured interval. This represents an upper limit of error that should never be exceeded, not the probable error, which would be one fourth as large.

\section{Method of Measurement}

The lens under test is placed in the chuck of the nodal slide assembly of the optical bench. The nodal slide assembly is moved along the ways of the optical bench until the image of a collimated source formed by the lens under test is viewed in the microscope of the optical bench. The reticle used in the collimator consists of a vertical and horizontal line at right angles to each other forming a cross. The nodal slide assembly is now adjusted by the lead screw of the optical bench until the image formed by the lens of the collimated beam proceeding from the illuminated reticle is in sharp focus. The biprism is now inserted close to the face of the lens under test between the lens and the collimated beam from the illuminated reticle. This is illustrated in figure 3 . The image of the reticle formed by the lens under test is split by the biprism into two images which are viewed in the ocular of the viewing microscope. Figure 4 is a photograph of the two split images as seen through the microscope. Care must be exercised in the orientation of the biprism to achieve maximum displacement of the split images. This orientation is produced by rotating the biprism within its cell about its optical axis until the two horizontal lines of the viewed images, if continued, would appear as one continuous line. The horizontal crosshair in the viewing microscope is used as a guide in making this adjustment. The biprism has no spherical refracting power so the position of best focus is not changed by the introduction of the biprism. The viewing microscope is equipped with a lateral adjustment and scale that allows measurements to be made to within $\pm 1 \mu$. The vertical arm of the crosshair in the microscope is brought into coincidence with the vertical line in the image of one of the two images formed by the biprism and lens by the lateral adjustment of the viewing microscope. A reading $R_{1}$ is taken of this position on the lateral scale of the microscope. The microscope is now traversed laterally until the vertical line in the crosshair of the microscope is in coincidence with the vertical line in the second image of the cross and a reading $R_{2}$ is taken on the lateral scale of the viewing microscope. Readings $R_{1}$ and $R_{2}$ are successively taken until an average of at least five readings of each position has been obtained. The focal length can then be determined from the following relation:

$$
D=R_{1 a}-R_{2 a}=2 f \tan \theta
$$




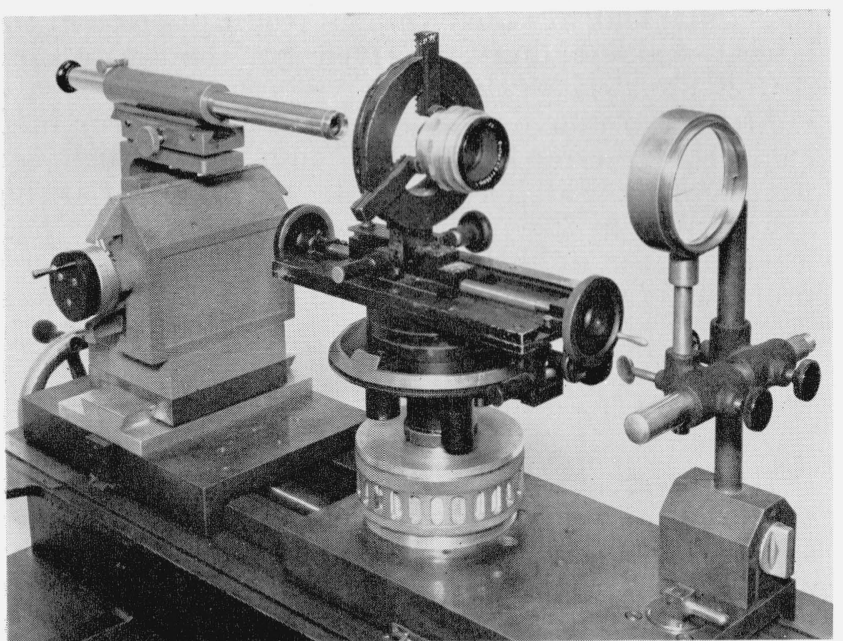

Figure 3. The briprism is inserted close to the face of the lens under test, between the lens and the collimated beam from the illuminated reticle.

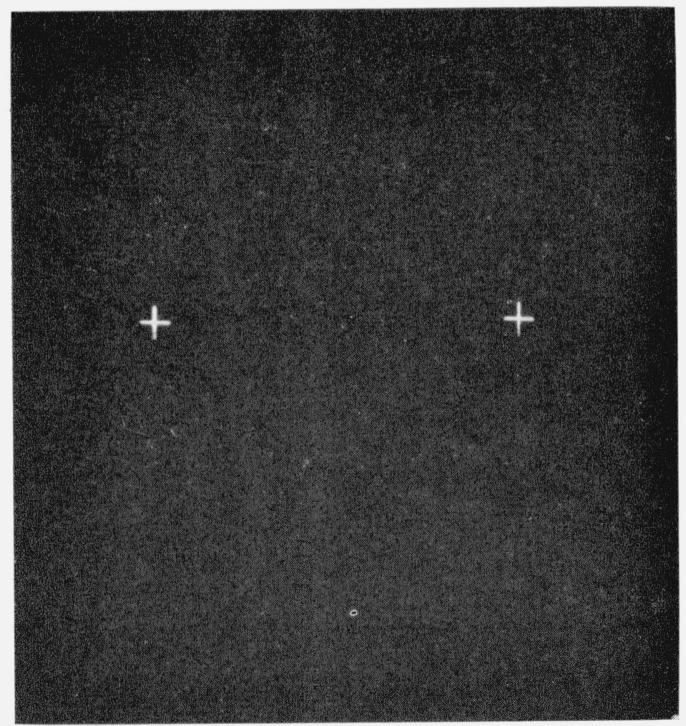

FIGURE 4. A photograph of the two split images as seen through the microscope.

or

$$
f=0.5 D \cot \theta
$$

where $D=R_{1 a}-R_{2 a}$ or the difference of the averages of the lateral scale measurements of $R_{1}$ and $R_{2}$. The quantity $0.5 \cot \theta$ is a constant of the instrument. Hence the value of the equivalent focal length may be obtained directly from the formula,

$$
f=27.81 D \text {. }
$$

\section{Precision Optical Bench}

The operation of the precision optical bench has been discussed in a previous paper. ${ }^{3}$ It may be mentioned here that the lens under test by the optical bench method of measurement is subjected to a number of critical time-consuming adjustments before any measurements are made. Depending upon the physical size of the lens and the focal length as much as two hours time is needed for adjustment of the lens on the precision optical bench.

\section{Results of Measurement}

The following table contains the results of measurements made on a number of lenses of varying focal lengths.

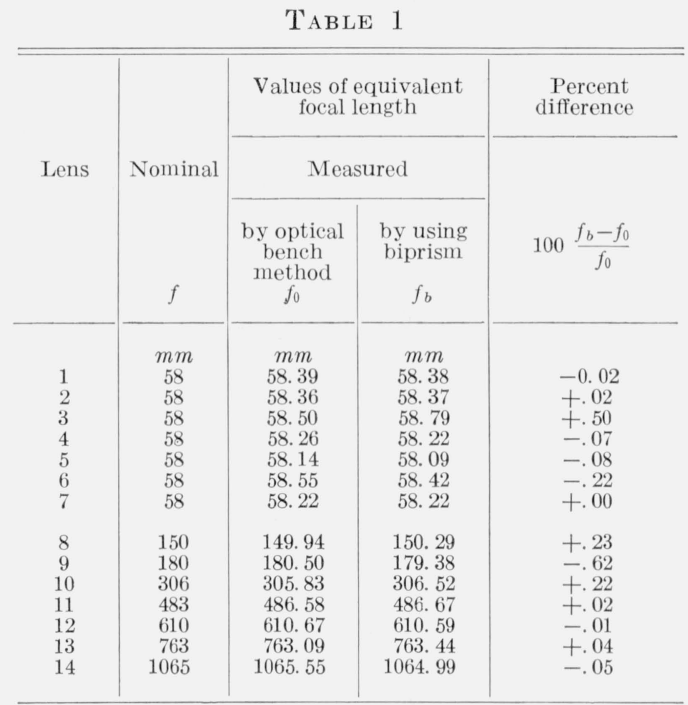

Average deviation $\pm 0.15 \%$

Improper orientation of the prism may lead to error, but even this is unlikely to lead to serious error as long as the prism axis does not deviate from normality with the ways of the measuring microscope by an amount exceeding \pm 2.5 degrees. A misorientation of this magnitude is not likely to occur as the splitting of the horizontal arm of the cross would immediately make manifest this type of error.

\section{Limitations in Measurements}

The maximum travel of the viewing microscope available for the measurement of $R^{1}$ and $R^{2}$ was 50 $\mathrm{mm}$. With the biprism whose angle of deviation being 2.059 degrees, the maximum equivalent focal length that can be determined is $1390 \mathrm{~mm}$. Longer focal lengths can be determined by decreasing the angle of deviation in the biprism or by increasing the lateral travel of the viewing microscope. Care must

3 F. O. Washer, W. R. Darling, Factors affecting the accuracy of distortion (1959). 
be exercised in using a prism of this nature, one must use a reasonably monochromatic light source, and the initial calibration of the prism must be made with near monochromatic light of approximately the same wavelength as that which will be used in the focal length determination.

Upon examination of the results of measurements obtained in the experimental data the focal length determinations by the biprism method were found to be biased. The values reported in table 1 using the biprism method were adjusted in the amount of -0.07 percent. A 0.07 percent change in the focal length amounts to approximately 5 sec change in deviation of the biprism, this is well within the tolerance of the measurement of the biprism. With the deviation from the average not greater than \pm 0.15 percent one can be confident that a focal length determination by means of the biprism is very unlikely to be error by as much as 1 percent.

The focal length determinations in table 1 were made with a Wratten No. 73 filter with an effective wavelength of approximately $575 \mathrm{~m} \mu$.

\section{Sources of Error}

Since $f=0.5 D \cot \theta$, error can be produced by errors in $D$ and by errors in $\theta$. Assuming $\theta$ to be without error, from the relation in eq (1) $0.5 D \cot \theta$ is a constant equal to 27.81. Therefore the error in the equivalent focal length as a result of error in the lateral scale readings is given by the expression: $\Delta f=27.81 \Delta D$, eq $(2)$.

From the above it is clear that a $2 \mu$ error in $D$ leads to an error of $\pm 0.055 \mathrm{~mm}$ in $f$ which can usually be considered negligible. Errors in $f$ arising from errors in the measured value of $\theta$ can be neglected as it is unlikely to exceed \pm 0.2 percent for a probable error of $6 \mathrm{sec}$ in an angle of 2.059 degrees.

\section{Discussion}

It is evident from the information contained in the foregoing sections that the biprism method is a satisfactory means for quickly determining the equivalent focal length of flat field lenses with an error that does not exceed \pm 1 percent. It is also pointed out that a nodal slide assembly is not a necessity in making the focal length determination. Any method that allows the lens to be held firmly and in a reasonable alinement with the collimated incident beam is sufficient.

Acknowledgment is made to F. E. Washer and R. E. Stephens for assistance and suggestions in the preparation of this paper.

(Paper 66C4-106) 